\title{
Therapeutic Efficacy of the Modified Epley Maneuver With a Pillow Under the Shoulders
}

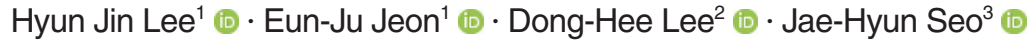 \\ ${ }^{\prime}$ Department of Otorhinolaryngology-Head and Neck Surgery, Incheon St. Mary's Hospital, College of Medicine, The Catholic University of \\ Korea, Incheon; ${ }^{2}$ Department of Otorhinolaryngology-Head and Neck Surgery, Uijeongbu St. Mary's Hospital, College of Medicine, The Catholic \\ University of Korea, Uijeongbu; ${ }^{3}$ Department of Otolaryngology-Head and Neck Surgery, Seoul St. Mary's Hospital, College of Medicine,
}

The Catholic University of Korea, Seoul, Korea

Objectives. Canalolithiasis of the posterior semicircular canal (PC) is the most common reason for benign paroxysmal positional vertigo (BPPV). If a patient is diagnosed with PC-BPPV through the Dix-Hallpike test, the modified Epley maneuver (mEpley) is used as the gold standard treatment. To reduce the discomfort of the standard mEpley, we proposed placing a pillow under the shoulders during this therapeutic maneuver.The purpose of this study was to determine whether the therapeutic efficacy of the mEpley with a pillow under the shoulders (mEpley-PS) was comparable to that of the standard mEpley.

Methods. A randomized controlled study at three academic referral hospitals was conducted in compliance with the CONSORT statement. Patients who were diagnosed with PC-BPPV through the Dix-Hallpike test were randomly assigned to groups A or B. Patients in groups A and B were treated with the standard mEpley and mEpley-PS, respectively. The resolution of vertigo and nystagmus on the Dix-Hallpike test at a 1-week follow-up after treatment was the main outcome measurement to assess the efficacy of treatment.

Results. Forty-one patients diagnosed with PC-BPPV were enrolled in this study. Twenty-one patients were assigned to group A and 20 were assigned to group B. The success rate at 1 week after treatment was $85.7 \%$ in group A and $80.0 \%$ in group B. There was no statistically significant difference between the two groups $(P=0.697)$.

Conclusion. The therapeutic efficacy of the mEpley-PS was comparable to that of the standard mEpley. The use of the pillow modification may be an excellent alternative if a patient cannot tolerate the head-hanging position, and it is helpful for patients who have anxiety about the head-hanging position. The mEpley-PS can be performed on a bed with or without a headboard. It is both a patient-friendly and a clinician-friendly maneuver.

Keywords. Benign Paroxysmal PositionalVertigo; Semicircular Canals; Epley Maneuver

\section{INTRODUCTION}

Benign paroxysmal positional vertigo (BPPV) is characterized by short, repetitive episodes of vertigo and characteristic nystag-

\footnotetext{
- Received November 8, 2019

Revised November 30, 2019

Accepted December 6, 2019

- Corresponding author: Jae-Hyun Seo

Department of Otolaryngology-Head and Neck Surgery, Seoul St. Mary's

Hospital, College of Medicine, The Catholic University of Korea,

222 Banpo-daero, Seocho-gu, Seoul 06591, Korea

Tel: +82-2-2258-6210, Fax: +82-50-4411-7964

E-mail: revivalseo@catholic.ac.kr
}

mus induced by positional changes. BPPV can occur in all three semicircular canals, with the posterior semicircular canal (PC) being most frequently affected [1,2]. Clinicians should confirm the diagnosis of posterior semicircular canal BPPV (PC-BPPV) by performing the Dix-Hallpike (DH) test [3]. During the DH test, the clinician moves the head of the patient through a set of specified positions to elicit the expected characteristic nystagmus of PC-BPPV [4].

The canalith repositioning maneuver (CRM), which is the treatment of choice for PC-BPPV, can be performed in an outpatient setting $[5,6]$. It is also called the "Epley maneuver," as it was first described by Epley [7] in 1992 and modified afterward.

Copyright $\odot 2020$ by Korean Society of Otorhinolaryngology-Head and Neck Surgery.

This is an open-access article distributed under the terms of the Creative Commons Attribution Non-Commercial License (https://creativecommons.org/licenses/by-nc/4.0)

which permits unrestricted non-commercial use, distribution, and reproduction in any medium, provided the original work is properly cited. 


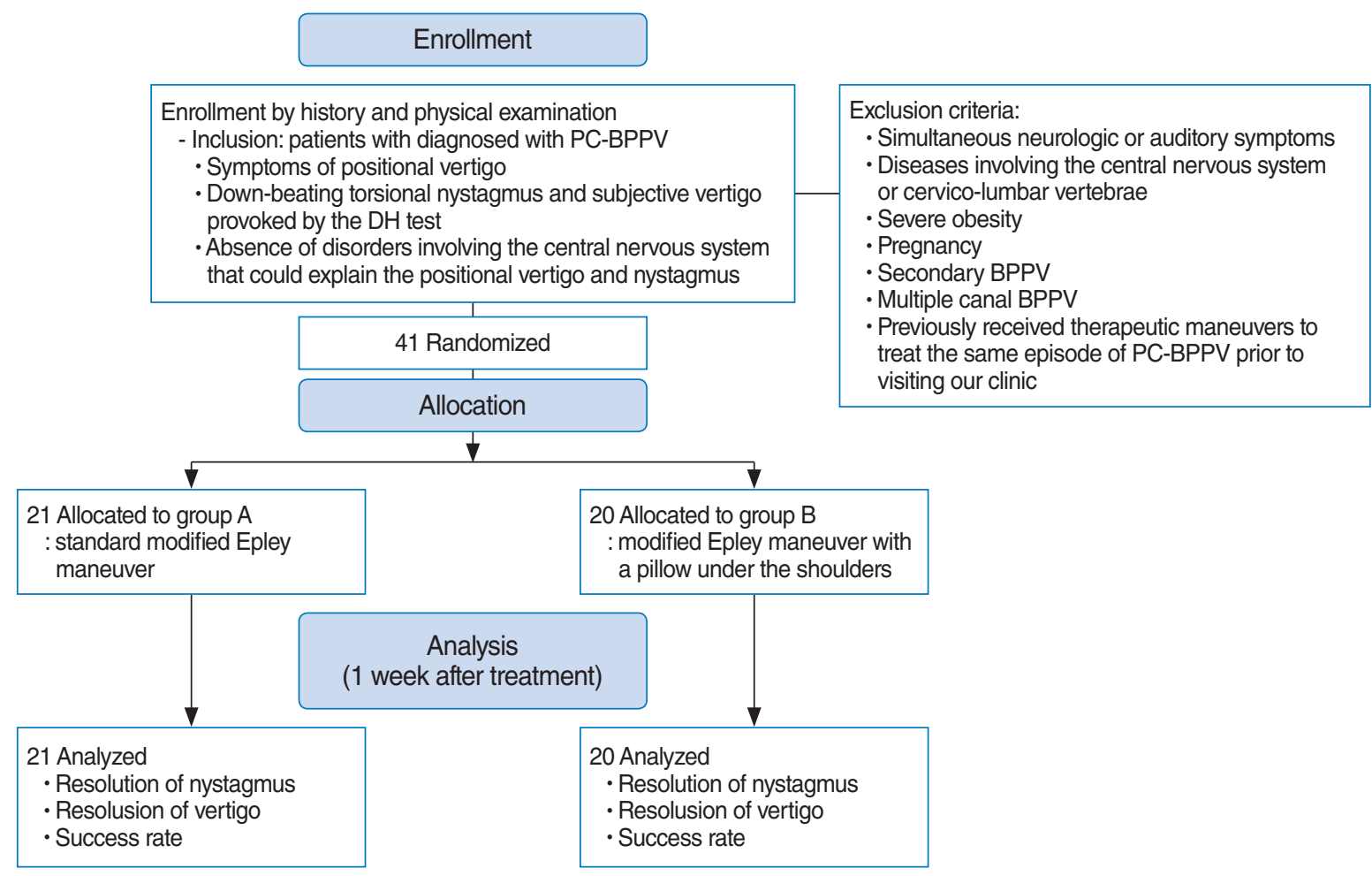

Fig. 1. Study protocol. Group A received the standard modified Epley maneuver and group B received the modified Epley maneuver with a pillow under the shoulders. The patients were treated with one of the two modified Epley maneuvers at the first visit and then visited the clinic 7 days after treatment for follow-up. PC, posterior semicircular canal; BPPV, benign paroxysmal positional vertigo; DH, Dix-Hallpike.

Although the modified Epley maneuver (mEpley) is known to be the best way to treat PC-BPPV, it has several disadvantages and limitations because of the head hanging position. In order to resolve these issues, the authors designed a further modification of the mEpley with a pillow under the shoulders (mEpleyPS). In the mEpley-PS technique, a dense foam pillow is placed under the patient's shoulders during the standard mEpley procedure, which allows the head to lay on the bed surface while the neck extends and rotates, providing comfort and stability to the patient throughout the procedure. The aim of this study was to investigate the therapeutic efficacy of the mEpley-PS in treating PC-BPPV compared to the standard mEpley.

\section{H I G H L I G G H T S}

- The therapeutic efficacy of the modified Epley maneuver with a pillow under the shoulders was comparable to that of the standard modified Epley maneuver.

- The pillow modification can be helpful for patients who have anxiety about the head-hanging position.

- The modified Epley maneuver with a pillow under the shoulders can reduce the strength and physical burden placed on clinicians because they no longer need to hold onto the patients' head.

\section{MATERIALS AND METHODS}

This study protocol was in compliance with the CONSORT 2010 statement for reporting the randomized controlled trial, and the trial was conducted according to the Declaration of Helsinki and all its revisions. It was approved by the Institutional Review Board of the CMC Clinical Research Coordinating Center (IRB No. XC13OIMI0053H). All patients provided written informed consent to participate in the trial.

\section{Patients}

This study was conducted at three referral academic hospitals between July and November of 2013. This study was a multicenter, prospective, randomized, single-blinded, and controlled clinical study. The patients who visited the referral academic hospitals for repeated issues of vertigo of brief periods induced by head movement were enrolled in this study. All patients who participated in this study fulfilled the following condition: (1) symptoms of positional vertigo, (2) down-beating torsional nystagmus and subjective vertigo provoked by the DH test, and (3) absence of disorders involving the central nervous system that could explain the positional vertigo and nystagmus. Patients who had simultaneous neurologic or auditory symptoms, diseases involving the central nervous system or cervico-lumbar vertebrae, exhibited severe obesity, were pregnant, and exhibit- 
ed secondary BPPV after head trauma or combined lateral canal BPPV were also excluded from this study. In addition, patients who have been received therapeutic maneuvers to treat the same episode of PC-BPPV prior to visiting our clinic were excluded.

\section{Experiment protocol}

Among the enrolled patients, only patients diagnosed with PCBPPV through the DH test participated in the study. The diagnostic criterion of PC-BPPV was the presence of both characteristic nystagmus and subjective vertigo induced by the $\mathrm{DH}$ test. Using a random table, the patients were assigned to either groups A or B. Afterwards, standard mEpley and mEpley-PS were performed for groups A and B, respectively (Fig. 1). All the treatments were conducted by experienced otolaryngologists. No special precautions or vestibular suppressant medications were prescribed after the procedure. Seven days after the treatment, the DH test was performed at an outpatient clinic to eval-

mEpley


Fig. 2. Difference in the head and neck positions between the standard modified Epley maneuver (mEpley) and the mEpley with a pillow under the shoulders (mEpley-PS). uate the therapeutic results of CRM. It was considered as success if nystagmus was not induced and the patient did not experience subjective vertigo during the $\mathrm{DH}$ test. If either nystagmus or vertigo symptoms were reported, it was considered a failure. The patients who exhibited treatment failure at the 1-week follow-up were followed up weekly, re-evaluated, and given individual treatments. Subsequent treatment results were not included in the analysis for the short-term efficacy of mEpley-PS in this study.

\section{Method of the mEpley-PS}

Standard mEpley is performed as stated in clinical practice guideline of BPPV [3]. Basically, mEpley-PS is performed in the same manner as standard mEpley, but a pillow is placed under the patient's shoulders, allowing for the extended head to rest on the bed surface (Fig. 2). Each step of the detailed method of Epley-PS is showed in Fig. 3. A dense foam pillow $10 \mathrm{~cm}$ in thickness was used for mEpley-PS. In a sitting position, the clinician determined the location of the patient's shoulders on the bed and placed a pillow to the estimated location. During mEpley-PS, the patient lays his or her shoulders on the pillow with the neck extending over the pillow and places his or her occiput on the surface of the bed naturally. With this modified maneuver, the examiner only needs to guide the direction and angle of the patient's movement and does not need to hold the patient's head up. Since the examiner does not hold up the patient's head in the air during the entire time of the maneuver, he or she can reduce the physical strain on his or her arms and back and focus on observing the patient's eye movements.

\section{Statistical analysis}

The chi-square test and Mann-Whitney $U$-test were used to compare the demographic data of the two groups. The chisquare test was used to compare the treatment success rate of the two groups. Statistical analysis was performed using SPSS

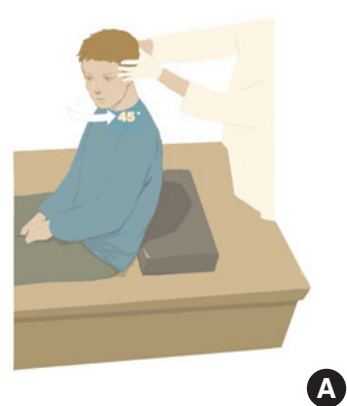

A.

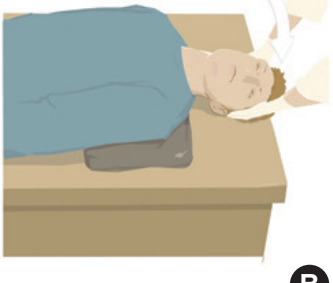

B



C
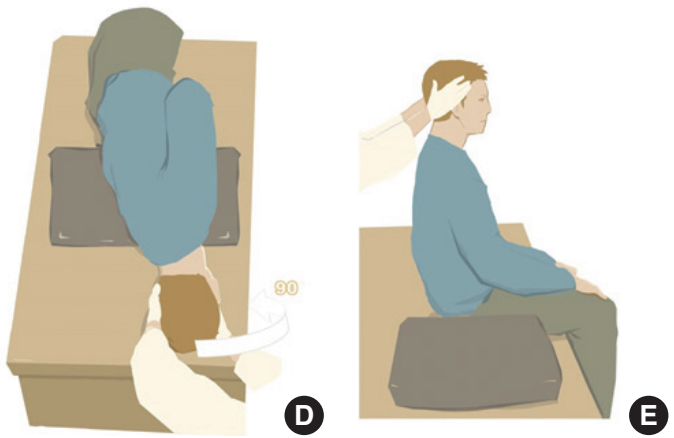

Fig. 3. Modified Epley maneuver with a pillow under the shoulders. (A) Ask the patient to sit upright on an examination table, and rotate the patient's head at a $45^{\circ}$ angle towards the affected side. (B) Quickly push the patient backward: the patient's upper body and head are reclined backward with the head extended $20^{\circ}-30^{\circ}$ below the pillow, resulting in a natural neck extension with the occiput resting on the surface of the table for 30 seconds. (C) Rotate the patient's head $90^{\circ}$ in the opposite direction, stably supporting it with the table surface. The examiner only guides the direction and angle of the patient's position for 30 seconds, until the dizziness stops. (D) Rotate the patient in the same direction another $90^{\circ}$, with the unaffected side facing down. The examiner holds the person in this position for 30 seconds, until his or her dizziness stops. (E) Finally, the examiner brings the patient back up to a sitting position. 
Table 1. Demographic data of the participants

\begin{tabular}{lccc}
\hline Variable & Group A & Group B & $P$-value \\
\hline No. of participants & 21 & 20 & - \\
Age (yr) & $53.2 \pm 11.2$ & $54.9 \pm 9.5$ & 0.603 \\
Sex (male:female) & $7: 14$ & $7: 13$ & 1.000 \\
Affected side (right:left) & $13: 8$ & $18: 2$ & - \\
Duration (day) & $8.14 \pm 10.07$ & $11.48 \pm 15.32$ & 0.204 \\
\hline
\end{tabular}

Values are presented as mean \pm standard deviation.

Group A, standard modified Epley maneuver; Group B, modified Epley maneuver with a pillow under the shoulders.

a) Interval between the onset of symptoms and treatment.

ver. 18.0 (SPSS Inc., Chicago, IL, USA). All tests were two-tailed and a $P$-value of $<0.05$ was considered significant.

\section{RESULTS}

After considering the inclusion and exclusion criteria and performing the DH test, 41 patients with PC-BPPV were enrolled in this study (Fig. 1). Twenty-one patients were assigned to group A and 20 patients were assigned to group B by random table allocation. The average age of the patients was $54.1 \pm 10.4$ years, with 14 men and 27 women. There were 31 cases of PC-BPPV of the right ear and 10 in the left ear. The demographic data of both groups are listed in Table 1 .

No statistically significant differences were found between the groups in terms of age, sex, laterality, or the interval between the onset of symptoms and the treatment (Table 1). The treatment success rate at 1 week after treatment was $85.7 \%$ (18 of 21 ) in group A and $80.0 \%$ (16 of 20 ) in group B (Table 2 ). The treatment success rate was not significantly different between the two groups $(P=0.697)$.

\section{DISCUSSION}

The Epley maneuver was introduced by Epley in 1992. In 1993, Parnes and Price-Jones [8] proposed the mEpley, which is performed through a single maneuver without the use of sedatives or vibrators. Since then, this mEpley maneuver has been considered the gold standard for the treatment of PC-BPPV [5,9]. However, several side effects related to the standard mEpley have been reported, such as a falling sensation, anxiety, and discomfort [10]. According to a clinical practice guideline, minor side effects such as nausea or imbalance may be associated with the standard mEpley, and these side effects might lead patients to be hesitant to undergo repeated maneuvers and an extended treatment period [11]. From the clinician's standpoint, rotating the patient's head below the table while holding it in the air is strenuous and can fatigue the clinician's arms and back. Recently, the authors reported that a modified DH test with the PS po-
Table 2. Treatment success rate

\begin{tabular}{lccc}
\hline Variable & Group A & Group B & $P$-value \\
\hline Success & 18 & 16 & - \\
Failure & 3 & 4 & - \\
Success rate (\%) & 85.7 & 80.0 & 0.697 \\
\hline
\end{tabular}

Group A, standard modified Epley maneuver; Group B, modified Epley maneuver with a pillow under the shoulders.

sition, proposed in order to resolve similar issues in the standard DH test, showed equivalent diagnostic efficacy to that of the standard test [12]. Placing the patient's head above the bed could provide a sense of stability and comfort during the procedure. The physical burden of the clinicians during the procedure is also reduced. PC-BPPV patients diagnosed through the modified $\mathrm{DH}$ test with the PS position also underwent the mEpley for treatment, as was the case with patients diagnosed with the standard DH test. It would make sense for the patients diagnosed with PC-BPPV through the modified DH test in the PS position to be treated with the mEpley-PS, which is performed in the exact same position. Therefore, it was necessary to verify the therapeutic effect of the mEpley in the PS position.

Since many BPPV patients visit the hospital through an emergency center where the beds are usually equipped with a headboard, it is common to test and treat BPPV patients in the PS position in clinical settings. In addition, the internet and YouTube also provide easy access to self-repositioning maneuvers or home Epley maneuvers in similar positions. Although many clinicians have been performing the mEpley with the patient's head placed on a bed, and self-repositioning maneuvers on a bed are becoming widespread, there have been no reports on the treatment results. In this randomized controlled study, we compared the treatment results of the mEpley-PS with that of the standard mEpley and found that there was no significant difference between the treatment results of both tests $(80.0 \%$ and $85.7 \%$, respectively). The short-term treatment results of the mEpley-PS were slightly poorer than those of the standard $\mathrm{mE}$ pley, but the results were statistically comparable.

Although it is assumed that treatment with the head on the table will make patients more comfortable, a major limitation of our study is that we did not evaluate patients' subjective ratings such as discomfort, pain, and anxiety during each maneuver. It also would have been better if we had been able to scientifically analyze the difference in the comfort that the clinicians actually felt during the examinations. Minor limitations of our study include its relatively small sample size and short-term follow-up period, which may have biased the results. Despite these shortcomings, our randomized controlled study demonstrated that the mEpley-PS had the same therapeutic efficacy as the standard mEpley, which was performed while the patient's head was hanging in the air. Our maneuver may be an alternative if a patient cannot tolerate the head-hanging position. It may also be helpful for nervous patients, who experience vertigo from the 
head positioning and complain of severe anxiety regarding the floating head position. This maneuver can reduce the physical burden placed on clinicians because they no longer need to hold on the patients' head. Since our maneuver does not require that the patient's head be hanging off the bed, it can be performed on beds with or without headboards.

The therapeutic efficacy of the mEpley-PS was comparable to that of the standard mEpley. The use of the pillow modification may be an excellent alternative if the head-hanging position is not an option for a patient and can be helpful for patients who have anxiety regarding the head-hanging position. The mEpleyPS can be performed on a bed with or without a headboard, and it is both a patient-friendly and clinician-friendly maneuver.

\section{CONFLICT OF INTEREST}

No potential conflict of interest relevant to this article was reported.

\section{ACKNOWLEDGMENTS}

The authors wish to acknowledge the financial support of the Catholic Medical Center Research Foundation made in the program year of 2018.

\section{ORCID}

Hyun Jin Lee https://orcid.org/0000-0003-3826-8830

Eun-Ju Jeon https://orcid.org/0000-0003-4566-6802

Dong-Hee Lee https://orcid.org/0000-0002-0401-1183

Jae-Hyun Seo https://orcid.org/0000-0002-8443-8581

\section{AUTHOR CONTRIBUTIONS}

Conceptualization: EJJ, DHL. Data curation: JHS, HJL. Formal analysis: JHS. Methodology: EJJ, DHL. Project administration: EJJ, DHL. Visualization: JHS. Writing-original draft: HJL. Writing-review \& editing: EJJ, DHL, JHS.

\section{REFERENCES}

1. von Brevern M, Bertholon P, Brandt T, Fife T, Imai T, Nuti D, et al. Benign paroxysmal positional vertigo: diagnostic criteria consensus document of the Committee for the Classification of Vestibular Disorders of the Barany Society. Acta Otorrinolaringol Esp. 2017 NovDec;68(6):349-60.

2. Dix MR, Hallpike CS. The pathology symptomatology and diagnosis of certain common disorders of the vestibular system. Proc R Soc Med. 1952 Jun;45(6):341-54.

3. Bhattacharyya N, Gubbels SP, Schwartz SR, Edlow JA, El-Kashlan H, Fife T, et al. Clinical practice guideline: benign paroxysmal positional vertigo (update). Otolaryngol Head Neck Surg. 2017 Mar;156(3_ suppl):S1-47.

4. Furman JM, Cass SP. Benign paroxysmal positional vertigo. N Engl J Med. 1999 Nov;341(21):1590-6.

5. Fife TD, Iverson DJ, Lempert T, Furman JM, Baloh RW, Tusa RJ, et al. Practice parameter: therapies for benign paroxysmal positional vertigo (an evidence-based review): report of the Quality Standards Subcommittee of the American Academy of Neurology. Neurology. 2008 May;70(22):2067-74.

6. Korres SG, Balatsouras DG. Diagnostic, pathophysiologic, and therapeutic aspects of benign paroxysmal positional vertigo. Otolaryngol Head Neck Surg. 2004 Oct;131(4):438-44.

7. Epley JM.The canalith repositioning procedure: for treatment of benign paroxysmal positional vertigo. Otolaryngol Head Neck Surg. 1992 Sep;107(3):399-404.

8. Parnes LS, Price-Jones RG. Particle repositioning maneuver for benign paroxysmal positional vertigo. Ann Otol Rhinol Laryngol. 1993 May;102(5):325-31.

9. Hilton MP, Pinder DK. The Epley (canalith repositioning) manoeuvre for benign paroxysmal positional vertigo. Cochrane Database Syst Rev. 2014;(12):CD003162.

10. Uneri A. Falling sensation in patients who undergo the Epley maneuver: a retrospective study. Ear Nose Throat J. 2005 Feb;84(2):82-5.

11. Maas BD, BruintjesTD, van der Zaag-Loonen HJ,Winters SM, MasiusOlthof S, Colijn C, et al. Physical and emotional burden of the Epley maneuver in the elderly. Otol Neurotol. 2019 Sep;40(8):1082-7.

12. Jeon EJ, Lee DH, Park JM, Oh JH, Seo JH. The efficacy of a modified Dix-Hallpike test with a pillow under shoulders. J Vestib Res. 2019;29(4):197-203. 